\title{
A Method to Estimate the Range of Validity of the Thin Film Approximation for Dielectric Function Determination in Nanostructures
}

\author{
Jiangtao Zhu, Peter A. Crozier and James R. Anderson
}

School for Engineering of Matter, Transport and Energy, Arizona State University, Tempe, AZ, USA

The thin film approximation can be employed to simplify the relationship between the single scattering distribution in electron energy-loss spectrum (EELS) and the dielectric function. This leads to the conventional formulation of Kramers-Kronig analysis (KKA) to extract optical properties from EELS [1]. We have successfully employed this approach to determine the optical properties of large carbonaceous aerosol particles of relevance to climate change [2, 3]. For nanoparticle systems and nanoparticles aggregates, the relationship between the energy loss spectrum and the dielectric function can be greatly complicated by surface and interface effects. It is important to know when the thin film approximate breaks down for these nanoscale systems. Here we use spectrum imaging and model carbonaceous spheres to explore the influence of surface and interface plasmons (in these systems Cerenkov effects are negligible) on the accuracy of the dielectric function determined from conventional KKA.

Polystyrene latex nanospheres about $80 \mathrm{~nm}$ in diameter were purchased from TED PELLA, INC. USA and employed as precursors. They were stabilized and converted to amorphous carbonaceous spheres in situ by performing an electron beam shower in STEM mode over a large area for about 15 minutes. Energy-loss spectrum images of single, and double spheres aggregates (Fig. 1a and b) were collected on the transmission electron aberration-corrected microscope 1 (TEAM1) with a monochromator at $80 \mathrm{keV}$ at the National Center for Electron Microscopy. The full width at half maximum (FWHM) of the zero-loss peak was about $0.15-0.21 \mathrm{eV}$ and the collection semi-angle was $30 \mathrm{mrad}$. Each spectrum image was typically $40 \times 40$ pixels with collection time at each pixel of about 30 microseconds and the time for a whole spectrum image was about 6 mins to balance sample drift and signal-to-noise ratio. For the spectrum image, principal component analysis (PCA) bought from HREM Research Inc. was used to remove the noise by reconstruction the first 20 components and the zero-loss peak was removed using the reflected tail method. Fourier-log deconvolution was applied to remove the plural scattering. The codes for KKA were modified from Egerton's code [1] with normalization by thickness [3]. The thin film approximation was used to estimate the surface contributions.

The derived dielectric functions at a wavelength of $550 \mathrm{~nm}$ as a function of position across the spheres is shown in Fig. 1c and d. Both its real part $\varepsilon_{1}$ and imaginary part $\varepsilon_{2}$ remain constant in the center regions of the spheres as expected but show significant change at the surfaces and the interfaces of the spheres. The thicknesses $(T)$ at each location along the lines are also included to show the center of the spheres. The suitable range for the thin film approximation for an $80 \mathrm{~nm}$ sphere spans a range of almost $60 \mathrm{~nm}$ across the center of the spheres. Dielectric functions in 0-55 $\mathrm{eV}$ from the centers of spheres for single and double spheres are also shown in Fig. 1d. The consistency of the dielectric functions suggests that the thin film approximation is valid on data acquired from the center of carbonaceous spheres as small as $40 \mathrm{~nm}$. 
It would be valuable to extend this approach to other nanomaterials systems. For example, we will apply our techniques to study $\mathrm{TiO}_{2}$ nanoparticles/aggregates by monochromated EELS. In this case the Cerenkov effects must be considered but may be significantly depressed by the small particle size. Moreover, with our new monochromated NION SuperSTEM 100 at ASU with demonstrated energy resolution of $12 \mathrm{meV}$, we will try to extend our dielectric determination into the near infrared [4].

\section{References}

[1] R.F. Egerton in "Electron energy-loss spectroscopy in the electron microscope", (Springer, New York) p.245.

[2] J. Zhu, P.A. Crozier, J.R. Anderson, ibid, these proceedings.

[3] D.T.L. Alexander, P.A. Crozier, J.R. Anderson, Science 321 (2008), p. 833.

[4] The authors acknowledge support of the National Center for Electron Microscopy, and the National Institute of Standards and Technology under Award 60NANB10D022. The authors acknowledge John M.Cowley Center for High Resolution Microscopy at Arizona State University.
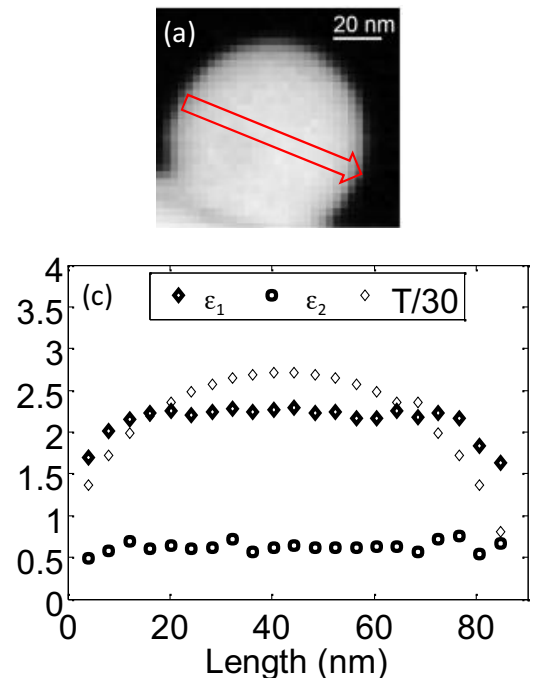
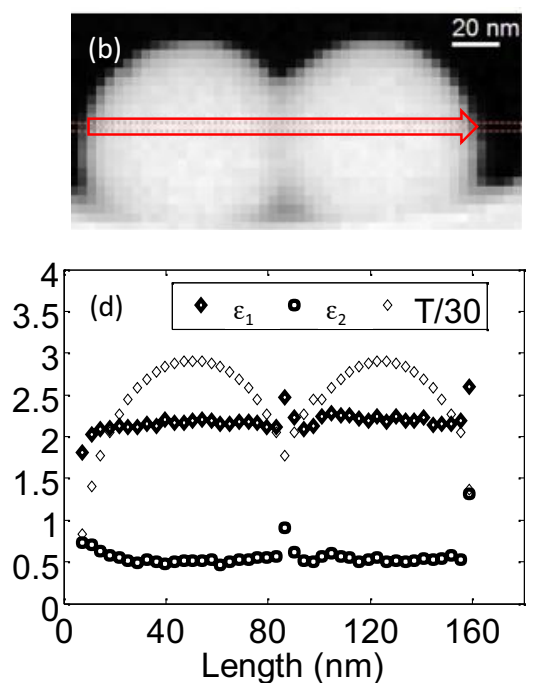

Figure1. The inelastic images of (a) single and (b) double carbonaceous spheres. The distributions of dielectric functions at $550 \mathrm{~nm}$ along the arrow lines in the spectrum images are shown in (c) for single sphere, and (d) for double spheres. $T$ is the thickness in $\mathrm{nm}$ at each point along the arrow lines (was divided by 30 in the curves). (e) The dielectric functions in the range of $0-55 \mathrm{eV}$ from the centers of the single and double spheres showing the consistency. 